\title{
LÍQUIDOS: UM ESTADO CRÍTICO
}

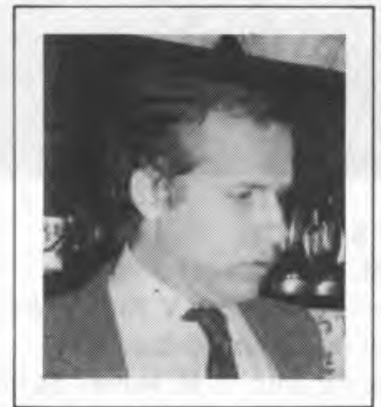

Jorge C. G. Calado

Departamento de Quimica-Fisica Instituto Superior Técnico 1000 Lisboa

\author{
Aos meus professores de Ciências Físico-químicas \\ que há 25 anos despertaram em mim. \\ o gosto pela beleza da verdade científica
}

\section{1 - Introdução}

O reconhecimento da existência de três estados da matéria distintos - sólido, líquido e gasoso - é tãc velho como a nossa civilização. Os quatro elementos de Empedocles de Agrigento (séc. VA.C.) são uma manifestação dessa realidade: a terra é sólida, a água é líquida e o ar é gasoso; o quarto elemento, o fogo, é a energia que permite transformar um estado noutro. No entanto, a explicação (quantitativa) das propriedades dos vários estados da matéria é muito mais recente e encontra-se na memória das quatro últimas gerações. Foi preciso esperar pelo século XIX, com os trabalhos de Gay-Lussac, Regnault, Andrews e van der Waals, entre outros, para se entender o comportamento dos gases reais; e foi já em pleno século XX que Bragg, Laue, Einstein, Born, Frenkel imprimiram a sua marca indelével ao desenvolvimento das teorias dos sólidos e suas propriedades.

Nada disto sucedeu por acaso. O século XIX, que viu soçobrar o neo-classicismo e esfumar-se o movimento romântico, trouxe-nos o impressionismo, a dissolução da realidade em impressões evanescentes. Turner cobriu as suas telas de fumos, vapores e nevoeiros e Monet via as catedrais ou as Houses of Parliament como neblinas impressionadas. Ruskin ambicionava engarrafar nuvens como o pai, que negociava em vinhos, engarrafava xerez. Até a técnica pontilista de um Seurat parece ser a contrapartida da visão mecacicista do estado gasoso, tal como nos é dada na teoria cinética ou na estatística de Boltzmann. Impressões fugidias, estados de alma, «chuveiro incessante de átomos inumeráveis», de que nos falava Virginia Woolf. Com o dobrar do século as impressões aglutinam-se e cristalizam em volumes. Na sequência de Cézanne, que nos ensinara a ver a realidade, incluindo a Natureza, em termos de formas simples como o cone, a esfera e o cubo, aparece o cubismo, as construções mecânicas de Léger. Na arte, tal como na ciência, a simplicidade do gás precede a do sólido.

Se o século XIX foi a era do estado gasoso, e o século $\mathrm{XX}$ a era do estado sólido, não parece que tenhamos de esperar pelo século XXI para ver resolvidos os problemas que entravam o desenvolvimento das teorias dos líquidos. Esta posição optimista foi resumida pelo Professor Rowlinson ao afirmar, na sua Liversidge Lecture de 1978, que "aaté há cerca de dez anos era vulgar ler-se em livros e artigos de revisão que pouco se sabia acerca da estrutura e propriedades de líquidos. ...Esta situação mudou agora dramaticamente.»' Referindo-nos a problemas específicos de misturas de liquidos, nós próprios tivemos também ocasião de escrever recentemente: «É hoje geralmente aceite que os problemas referentes à mecânica estatística de misturas líquidas de moléculas simples estão resolvidos. ...É possivel fazer predições bastante correctas das funções de excesso termodinâmicas de sistemas simples. $n^{2}$ Mesmo em 1973, ao fazer o ponto da situação da teoria de equilibrio de misturas líquidas, o Dr. McDonald afirmava: «Não é exagero dizer que o problema do cálculo das propriedades termodinâmicas de misturas simples está agora amplamente resolvido. ${ }^{3}$

Mas justificar-se-á um tal grau de optimismo, ou encontram-se os líquidos num estado critico de conhecimento, com avanço oscilante? E mesmo nos domínios em que tem sido possível fazer progressos notáveis não haverá coincidências fortuitas, com um «demónio» a jogar jogos de azar ou a fazer batota para nós ganharmos? Porque é que uma teoria relativamente rudimentar, como a chamada teoria de van der Waals na versão de um-fluído, produz resultados sensacionais, e uma versão mais depurada, mais próxima da realidade, como é a de dois-fluidos, não é mais do que um fracasso? São algumas destas contradições que vou desenvolver nesta lição, aproveitando ao mesmo tempo para apresentar alguns conceitos simples dạ mecânica estatística do estado líquido.

\section{2 - Tipos de Fluidos}

Conhece-se ou compreende-se uma substância quando é possivel prever quantitativamente as suas propriedades. Idealmente deveria ser viável explicar em que condições há mudança de fase, mas devido à complexidade do problema, tradicionalmente os vários estados são estudados em separado. As teorias de que nos servimos para explicar as propriedades do gelo não são as mesmas a que recorremos quando procuramos compreender os desvios à idealidade do vapor de água. No entanto, a substância é a mesma, e o conhecimento das característi- 
cas individuais da molécula, bem como da energia de interacção entre moléculas, deve conter os vários estados em que macroscopicamente a substância se pode apresentar. Como diz Ulrich, o protagonista de Der Mann ohne Eigenschaften (O Homem sem Qualidades) de Robert Musil, «este arquétipo de todos os liquidos (a água) não era, de modo algum, do ponto de vista físico, um liquido mas, consoante as circunstâncias, um corpo sólido, um líquido ou um gás. Em último lugar tudo se dissolvia num sistema de fórmulas que estavam de algum modo todas relacionadas umas com as outras. $n^{4}$

A previsão das propriedades duma substância começa com o estabelecimento dum modelo para a molécula, continua com o postular dum determinado potencial intermolecular e culmina com a aplicação dos métodos da mecânica estatística ao conjunto macroscópico de moléculas, de acordo com o diagrama seguinte

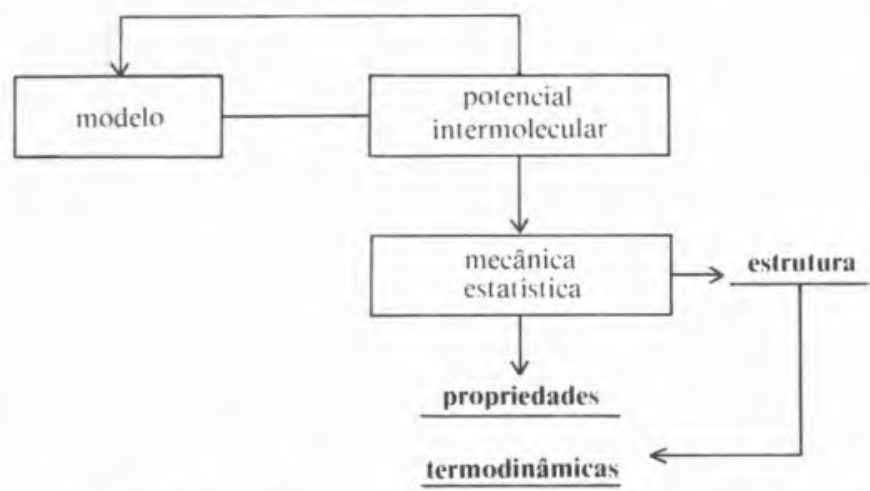

Considerando, por exemplo, o metano $\mathrm{CH}_{4}$, à partida temos de escolher um modelo para a molécula, isto é, se a tomamos como esférica dada a elevada simetria que a caracteriza, ou como constituida por um átomo de carbono e quatro de hidrogénio disıribuidos segundo um tetraedro; temos de decidir ainda se as distâncias entre átomos sâo fixas ou se levamos em conta a vibração. Esta escolha vai, de certo modo, influenciar a selecção dum potencial intermolecular adequado. $\mathrm{Na}$ primeira hipótese, da molécula esférica, o potencial é do tipo esfericamente simétrico, isto é, apenas depende da distância que separa as moléculas, u(r); na segunda, é lógico tomar-se uma interacção átomo-a-átomo, isto é, a interacção entre duas moléculas de metano é o resultado da interacção entre o átomo de carbono duma e os átomos de hidrogénio da outra $(\mathrm{C}-\mathrm{H})$, entre os carbonos de cada uma $(\mathrm{C}-\mathrm{C})$, os hidrogénios das duas $(\mathrm{H}-\mathrm{H})$, etc. Podemos ainda tomar a molécula como pontual ou com dimensões, rigida ou macia, com cerne (núcleo) ou sem cerne. A força de interacção (intermolecular) é dada por

$$
F(r)=-\frac{d u(r)}{d r}
$$

Quanto às técnicas da mecânica estatística, são métodos aproximados de cálculo de integrais (nomeadamente do chamado integral configuracional que envolve o conhecimento do potencial intermolecular) ou de resolução de equações integrais. Não há soluçôes exactas para os problemas da termodinâmica estatística de líquidos reais. Em princípio aqueles métodos fornecem quer a estrutura (o modo como as moléculas se empacotam umas em torno das outras), quer as propriedades termodinâmicas de equilibrio (ener- gia, densidade, pressão, compressibilidade, capacidade calorifica, etc.) ou de transporte (viscosidade, condutibilidade térmica, coeficientes de difusão) que, evidentemente, dependem dessa estrutura. Ora, se é certo que se têm produzido progressos notáveis na idealização e refinamento das técnicas da mecânica estatística, principalmente graças à utilização de computadores rápidos, não é menos certo que não se passou ainda dos primórdios do conhecimento do potencial intermolecular. Dominado que foi o problema das forças intermoleculares das moléculas esféricas ou quase-esfé ricas, hoje as atençôes concentram-se nas moléculas diatómicas como o azoto, o cloro, o monóxido de carbono. No entanto, para nenhuma destas espécies se conhece o potencial intermolecular com o pormenor com que é conhecido, por exemplo, para o árgon (ou para o cripton, ou para o xénon). Estas são, aliás, as únicas substâncias cujas propriedades podem ser previstas com base num único potencial intermolecular, aplicável ao gás, ao liquido e ao sólido.

Os progressos conseguidos no estado líquido sâo essencialmente matemáticos, de métodos de cálculo, mas assentam em modelos grosseiros e versōes ultra-simplificadas da energia potencial de interacção molecular.

Como disse o professor Andersen, «a relação entre a física dos liquidos e a matemática das nossas teorias está ainda muito pouco compreendida.."

Qualquer que seja o potencial, ele deve conter uma parte repulsiva de curto alcance ou cerne (a repulsão electrónica é responsável pela impenetrabilidade da matéria) e uma parte de longo alcance, tendendo para zero com a distância, a que se chama cauda. A estrutura e as propriedades dos liquidos dependem das caracteristicas destes dois componentes do potencial. Exemplo de potencial de longo alcance, com valores apreciáveis para disıâncias grandes, é o coulombiano, gerado pelas cargas eléctricas

$$
\mathrm{u}(\mathrm{r}) \propto \frac{1}{\mathrm{r}} \quad \text { grande alcance }
$$

O potencial gerado por um dipolo, que varia com $\mathrm{r}^{-3}$, é de alcance médio, enquanto que os potenciais que dependem de potências de ordem superior são de curto alcance

$$
u(r) \propto \frac{1}{6} \quad \text { curto alcance }
$$

A ligação por ponte de hidrogénio é outro exemplo de in teracção de curlo alcance.

Um aspecto típico de potencial intermolecular é o indicado na figura 1. Tal potencial é caracterizado por, pelo menos, dois parâmetros - por exemplo, uma energia (a profundidade do fosso, $-\varepsilon$ ) e uma extensão (a distância finita de separação para a qual a energia de interacção é nula $\sigma$, que é uma medida da dimensão molecular). Um exemplo muito utilizado nos cálculos é o do potencial de Lennard-Jones, dado por

$$
u(r)=4 \varepsilon\left\{\left(\frac{\sigma}{r}\right)^{12}-\left(\mid r^{-}\right)^{6}\right\}
$$

Embora os pormenores do potencial real não possam ser definidos por uma curva biparamétrica, o conhecimento de $\varepsilon$ e $\sigma$ dá-nos qualitativamente a essência do tipo de interacção. 


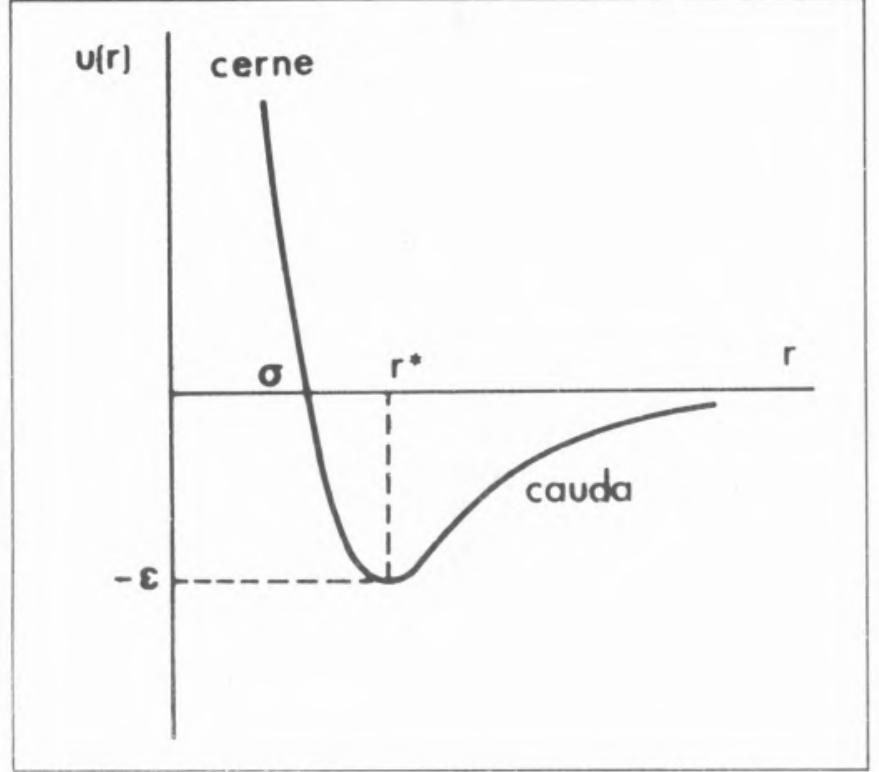

Fig. 1 -Potencial intermoleculat

Para efeitos de classificação $\varepsilon$ é comparado con kT $\sigma^{3} \operatorname{com} 1 / \rho$, onde $\rho$ é a densidade numérica (número de moléculas na unidade de volume)

$$
\rho=\frac{N}{V}
$$

$\rho \sigma^{3}<0.5$ caracteriza fluidos de densidade baixa, e $\varepsilon / \mathrm{kT}<1.5$ fluidos de interacção fraca. Temos assim os seguintes tipos de fluidos na classificação de Andersen .

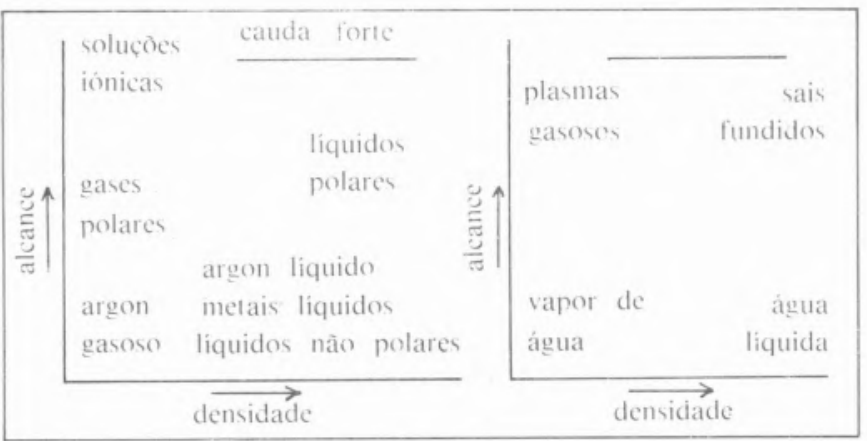

\section{3 - Fluidos Simples e a Ideia de van der Waals}

Tal como referimos atrás, os progressos conseguidos no estado liquido resumem-se ao estudo de liquidos simples, e mesmo assim utilizando formas grosseiras de potencial. Por moléculas simples entendem-se aquelas que interactuam segundo um potencial que é esfericamente simétrico e que não carecem de correcções quânticas. Trata-se, necessariamente, de moléculas pequenas em que os efeitos de estrutura são desprezáveis ou são arredondados. Os efeitos quânticos tornam-se importantes para moléculas pequenas e leves e de fraca interacção, e são medidos pelo parâmetro $\Lambda$

$$
\Lambda=\frac{h}{\sigma \sqrt{m \varepsilon}}
$$

Admite-se ainda, em geral, aditividade dos potenciais intermoleculares, isto é, a energia potencial de interacção total $\Phi$ é a soma das energias correspondentes a todos os pares possiveis

$$
\Phi={ }_{i<j}^{\Sigma} u_{i j}
$$

Na tabela 1 apresenta-se a lista de moléculas simples (hélio, néon, hidrogénio não estão incluidos devido às complicações quânticas).

Tabela 1. Moléculas Simples

$\begin{array}{ll}\text { esféricas } & \mathrm{Ar}, \mathrm{Kr}, \mathrm{Xe} \\ \text { quase-esféricas } & \mathrm{CH}_{4}, \mathrm{CF}_{4}, \mathrm{SF}_{6} \\ \text { diatómicas } & \mathrm{N}_{2}, \mathrm{O}_{2}, \mathrm{~F}_{2}, \mathrm{Cl}_{2}, \mathrm{Br} 2 \\ \text { polares } & \mathrm{CO}, \mathrm{NO}, \mathrm{HCl}, \mathrm{HBr} \\ \text { poliatómicas } & \mathrm{CO}_{2}, \mathrm{CS}_{2} \\ \text { hidrocarbonetos } & \mathrm{C}_{2} \mathrm{H}_{2}, \mathrm{C}_{2} \mathrm{H}_{4}, \mathrm{C}_{2} \mathrm{H}_{6}, \mathrm{C}_{2} \mathrm{H}_{8}\end{array}$

$\mathrm{Na}$ classificação de Andersen tais substâncias caracterizam-se por apresentar densidade elevada. interacção de curto ou médio alcance com cauda fraca e originam, em gerál, diagramas de equilíbrio simples, do tipo representado nas figuras 2 e 3 .

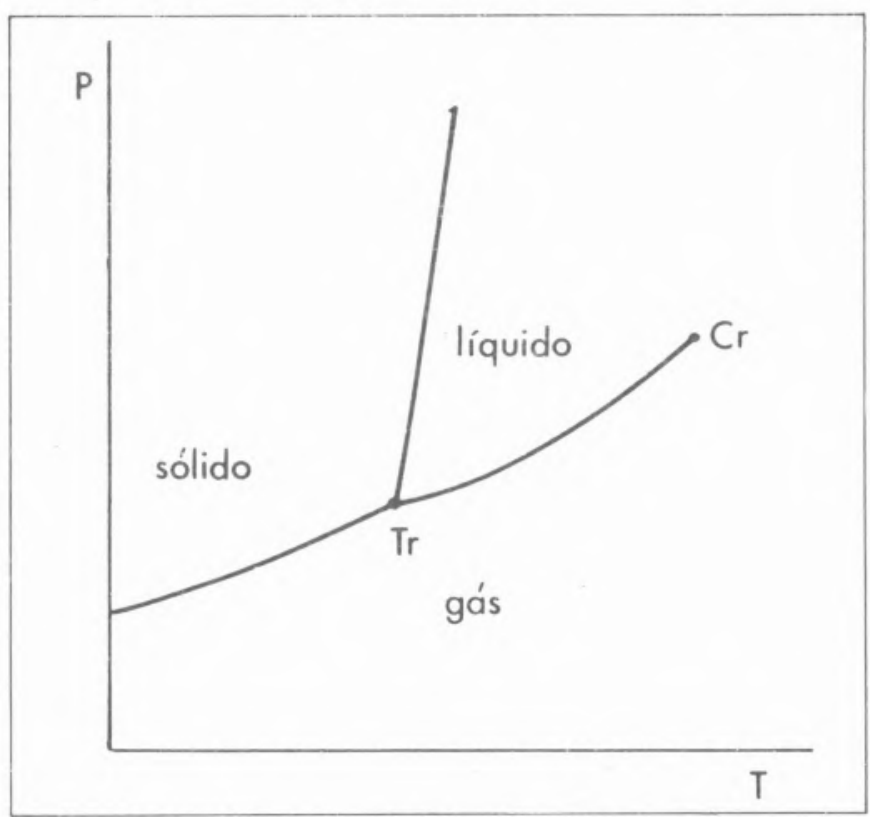

Fig. 2 - Diagrama de fases (p.T) para subsıâncias simples. Tr, ponıo ponto triplo; $\mathrm{Cr}$, ponto critico

O que permitiu o extraordinário avanço da física dos líquidos simples nesta década de setenta foi o renascimento da ideia, que vem de van der Waals, de que $\dot{e} a$ parte 'dura' do potencial repulsivo que determina a estrutura do líquido. Em linguagem corrente, a parte 'dura' do potencial (isto é, aquela que varia muito rapidamente com a distância) anda associada aquilo a que se chama a forma da molécula (o tamanho desta é uma consequência das forças repulsivas), de modo que a ideia de van der Waals também pode ser enunciada da seguinte maneira (Chandler): a forma das moléculas determina as correlações intermoleculares.

Deve notar-se, no entanto, que este conceito apenas se aplica a liquidos de densidades elevadas, isto é, em que $\rho \geq 2 \rho_{\mathrm{c}}$ (onde $\rho_{\mathrm{c}}$ é a denşidade numérica crítica; para termo de comparação observe-se que a densidade do liquido no ponto triplo é $\sim 3 \rho_{\mathrm{c}}$ ) e em que a parte do potencial que varia acentuadamente com a distância é a repulsiva $(\mathrm{vd}$. figura 1$)$.

$$
\left(r^{*}-\sigma\right) / r^{*} \leq 0.1
$$




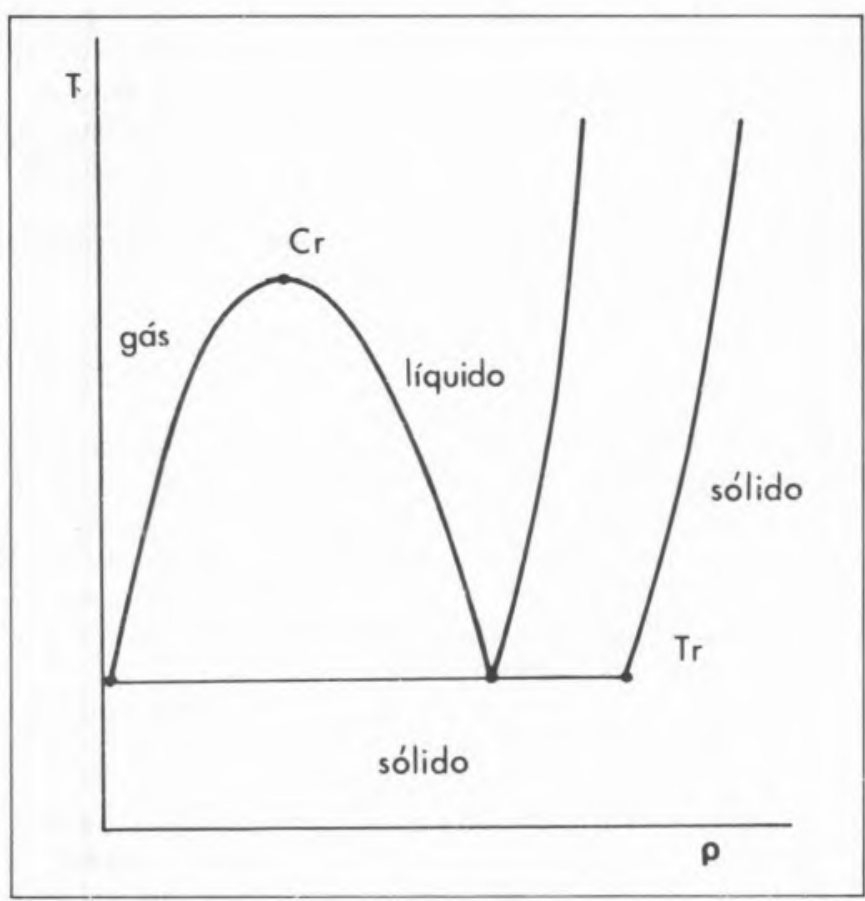

Fig. 3 - Diagrama de fases (T,p) para substâncias simples

Estas restrições fazem com que a ideia de van der Waals não se aplique nem a gases (mesmo densos) nem a líquidos associados como a água, mas ela tem, mesme assim, uma certa generalidade, cobrindo liquidos tão diferentes como o árgon, o azoto, o tetracloreto de carbono, benzeno e mesmo liquidos fortemente polares nào associados como 0 acetonitrilo $\mathrm{CH}_{2} \mathrm{CN}$.

A explicação da ideia de van der Waals em termos correntes é simples; com efeito, nos estados densos as moléculas estão bastante próximas umas das outras, com distâncias de separação médias da ordem de $r^{*}\left(\rho-3 \leq r^{*}\right)$ e qualquer pequena deslocação das moléculas causa

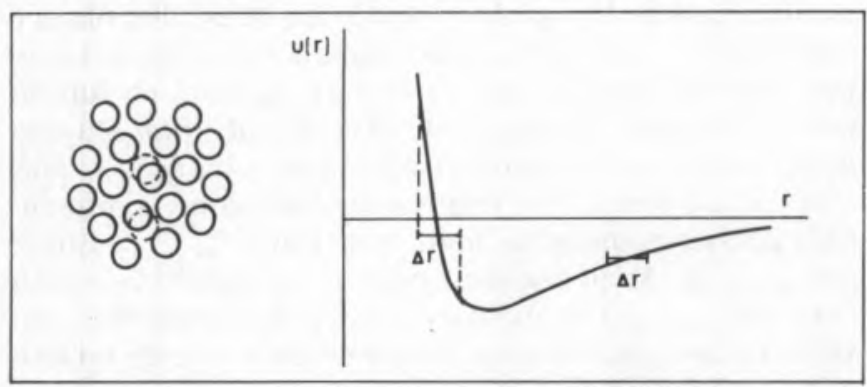

Fig. 4 - Variação da energia polencial repulsiva e atractiva com a mudança da distância de separação $\Delta r$

grandes variaçôes na energia potencial repulsiva, como se pode perceber pela figura 4 . Pelo contrário, a parte atractiva do potencial intermolecular varia pouco com a distância, resultando daqui que as forças atractivas, nomeadamente as de natureza electrostática (interacçōes dipolo-dipolo, dipolo-quadrupolo, quadrupolo-quadrupolo) desempenham um papel menor na estrutura do liquido. O seu efeito primordial é criarem um campo médio ou potencial de fundo uniforme que mantém estável o estado de elevada densidade que é o liquido a pressôes elevadas ( $\mathrm{tal}$ como os núcleos são estáveis graças às forças nucleares, apesar da repulsão electrostática dos protōes). Mas uma vez atingida a elevada densidade por obra da 'cola invisivel' que são as forças atractivas, a maneira como as moléculas se distribuem umas em torno das outras, o seu empacotamento, é determinada pelas forças repulsivas (forma e tamanho). É esta a base da teoria de Weeks, Chandler e Andersen ${ }^{7}$ (abreviadamente WCA) desenvolvida a partir de 1980. Segundo estas ideias a estrutura do árgon liquido é semelhante à das bolas de pingue pongue numa caixa, tal como a do anidrido carbónico liquido se assemelha à dos pregos numa lata e a do benzeno à dos 'corn-flakes' numa tijela (admitindo que os 'corn-flakes' têm forma hexagonal plana). Isto acontece porque todas estas moléculas podem ser razoavelmente representadas por corpos rígidos de dimensão. no entanto, para o bromo parece já não ser assim: Hsu, Chandler e Lowden mostraram que a relativamente grande separação de cargas na molécula, responsável pelo elevado momento quadrupolar, produz interaccões repulsivas especificas de curto alcance que impedem as moléculas de se aproximarem tanto quanto seria de esperar dada a sua constituição. ${ }^{\star}$ Outra excepção são os liquidos associados çom o cloreto de hidrogénio $\mathrm{HCl}$, em que a estrutura é determinada por forças atractivas de curto alcance (pont es de hidrogénio). Igualmente os sais fundidos, em que existem fortes força atractivas entre os iòes que variam rapidamente com a distância, não podem ser ıratados pelo modelo de van der Waals.

Quando se fala de corpos rigidos há, evidentemente. uma aproximação: as moléculas são mais ou menos duras mas não são infinitamente rígidas, e na prática ( que se faz é substituir $\sigma$ pelo diâmetro da esfera rigida equivalente $d$, com $d \backsim 1.02 \sigma$, como se indica na fi. gura 5

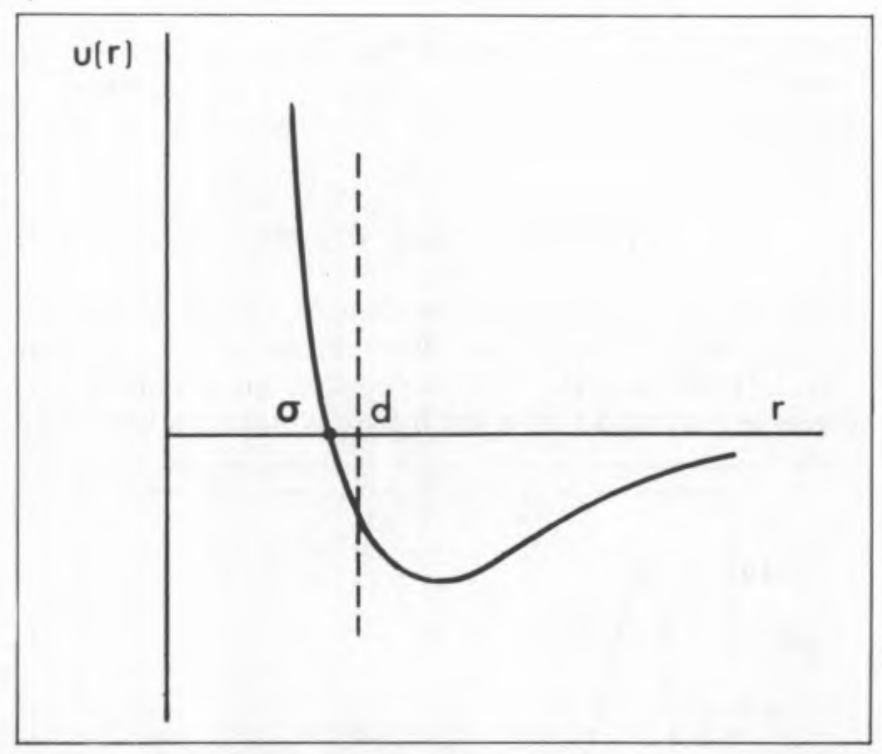

Fig. 5-Polencial real e diâmeıro da esfera rígida equivalente, d.

A extraordinária importância do modelo das esferas rigidas (que vem de vąn der Waals) no desenvolvimento da física dos liquidos revelou-se, em toda a sua beleza, com a descoberta de que um fluido de esferas rígidas (e também um fluido de discos rígidos, mas não um fluido de segmentos rígidos) pode dolidificar, o que mostra que o fenómeno da fusão-solidificação é uma consequência da parte repulsiva do potencial. Este resultado foi obtido por técnicas computacionais modernas, nomeadamente os métodos de Monte-Carlo e de Dinâmica Molecular ${ }^{9.10}$, e na figura 6 mostra-se o gráfico obtido por Hoover e Ree para esferas rígidas", com o patamar característico da mudança de estado (pressão constante). 


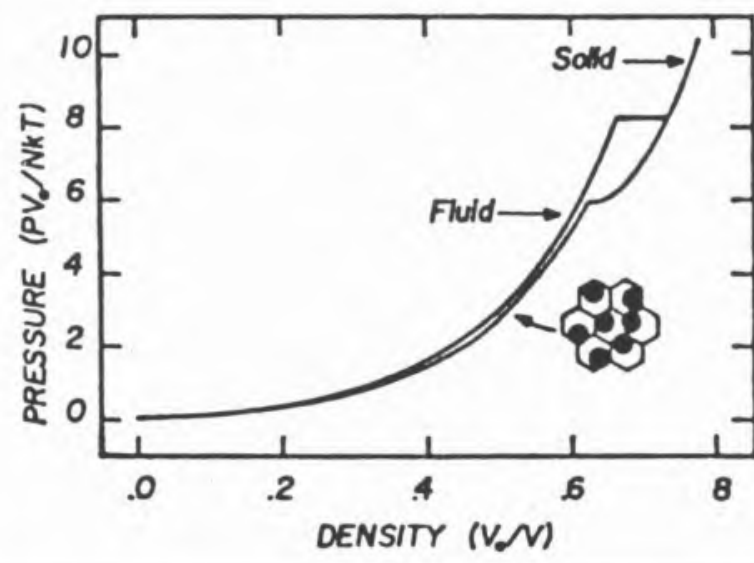

Fig. 6 - Equação de esıado para sistema de esferas rigidas, obtida por cálculos de Monte-Carlo (Ref. II)

Esıa figura é reproduzida, com aulorizacão, de Journal of Chemical Phvsics, Volume 48, pág. 36/4. ic 1968 by American Insrimute of Physics

\section{Estrutura e Propriedades Termodinâmicas}

A estrutura dum estado condensado é um conceito intuitivo, mas é também passivel duma descrição quanıitativa. Se se tratar dum estado isotrópico, a probabilidade $\Pi(1)$ de se encontrar uma molécula no elemento de volume $\mathrm{d} \tau$ em torno do ponto $(\mathrm{x}, \mathrm{j}, \mathrm{z})$ é proporcional a $\mathrm{d} \tau$ e dada por

$$
\Pi(1)=\frac{N}{V} \mathrm{~d} \tau=\rho \mathrm{d} \tau
$$

onde $\rho$ é a densidade numérica. De modo análogo a probabilidade $\Pi(1,2)$ de se encontrarem moléculas simulıaneamente em dois pontos à disıância $\mathrm{r}$ um dôn oulro é dada por

$$
\Pi(1,2)=\rho^{2} g(r) \quad d \tau_{1} d \tau_{2}
$$

onde $\mathrm{g}(\mathrm{r})$ é a função de distribuição radial. Podem-se ainda definir funções de disıribuição de $3 .^{\text {a }}$ ordem

$(1,2,3)$ envolvendo três moléculas, etc., mas o seu interesse é reduzido uma vez que se admite a aditividade

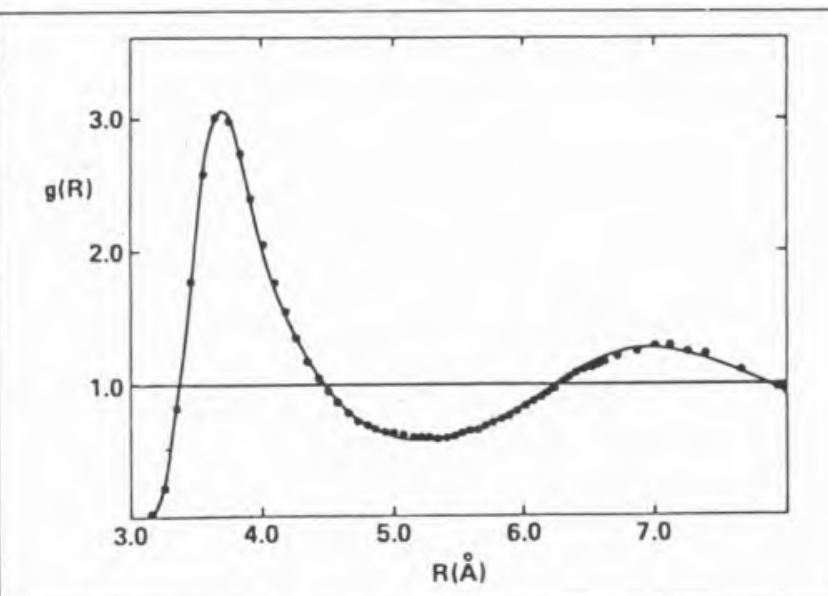

Fig. 7 - Funcầo de distribuição redial g(r) do argon liquido a $85 \mathrm{~K}$ Curva evperimental, obtida por difraccão de neturōes, e pontos calculados pelo método de Monte-Carlo (Ref. I0)

Esıa fieura è reproduzida, com aubrização, de Reviews of Modern Phvsies, Volume 48, pág. 590 (c) 1976 by American Physical Society dos potenciais intermoleculares $\left({ }^{7}\right)$, g(r), que para liquidos simples tem o aspecto representado na figura 7 , constitui uma descrição matemática da estrutura do fluído, isto é, é uma medida quantitativa dessa estrutura. A existência de picos pronunciados para distâncias curtas dá conta da ordem de curto alcance que caracteriza o liquido, ordem que se perde para longas dislâncias, quando $g(r)$ tende para o valor unidade. A função de distribuição radial mede também a densidade local em relação à densidade média, isıo é

$$
\mathrm{g}(\mathrm{r})=\frac{\rho(\mathrm{r})}{\rho}
$$

$\mathrm{g}(\mathrm{r})$ não depende apenas da distância $\mathrm{r}$; é tambémı uma funçào da temperatura e, mais acentuadamente, da densidade. Esıá ainda intimamente ligada à forma do potencial intermolecular - $g(r)$ anula-se para a disıancia minima de aproximação, e a maneira como cresce com r depende da dureza do cerne; $g(r)$ depende ainda da intensidade e alcance da cauda do potencial.

A função de distribuição radial representada na figura 7 é um exemplo típico de $\mathrm{g}(\mathrm{r})$ para um meio isotrópico, como é o caso de líquidos feitos de moléculas esféricas. No entanto, g(r) é também função da anisotropia do potencial, e um exemplo simples ajudará a esclarecer este ponto. Consideremos o caso do tetracloreto de carbono, $\mathrm{CCl}_{4}$. À temperatura ambiente e à pressão atmosférica a sua densidade é 1.595 , a que corresponde um volume molar de $96.5 \mathrm{~cm}^{3} \mathrm{~mol}^{-1}$. Isto significa que o volume ocupado por molécula é cerca de 160 $\mathrm{A}^{3}$, o que dá cerca de $5.4 \mathrm{~A}$ para a 'espessura' duma molécula no estado liquido. No entanto, recorrendo aos valores dos raios covalentes dos átomos de carbono e cloro chega-se à conclusão que a corpulência duma molécula de $\mathrm{CC}_{1}$, deve ser da ordem de $7 \mathrm{~A}$, de modo que o resultado anterior significa que no estado liquido há interpenetração das moléculas. Por outras palavras, o modelo das esferas rígidas não é aplicável ao tetracloreto de carbono (como não será aplicável ao metano), sendo necessário um esquema mais sofisticado que dê efectivamente conta da forma desta molécula. A escolha óbvia é o tetraedro, e isto introduz imediatamente anisot ropia no potencial intermolecular, e portanto também na funçâo de distribuição. A funçâo de distribuição total é agora uma funçào nào só duma distância de separação r, mas também da orientação relativa das moléculas, represenIada pelos conjuntos de ângulos de Euler, $\omega_{1}, \omega_{2}$, isto é, $\mathrm{g}\left(\mathrm{r}, \omega_{1}, \omega_{2}\right)$. Analogamente para o potencial intermolecular $\mathrm{u}\left(\mathrm{r}, \omega_{1}, \omega_{2}\right)$. O método clássico de tratamento consiste em desenvolver estas funçōes numa série de polinómios ortogonais. Por exemplo, para moléculas diatómicas a orientaçâo relativa é determinada por ırês ângulos $\theta_{\mathrm{i}}, \theta_{\mathrm{i}}$ e $\phi_{\mathrm{ii}}=\phi_{\mathrm{i}}-\phi_{\mathrm{i}}$, de modo que

$$
g\left(r, \theta_{i}, \theta_{j}, \phi_{i j}\right)=4 \pi \underset{\ell \ell^{\prime} m}{\sum} g_{\ell \ell ' m}(r) Y_{\ell m}\left(\theta_{i}, \phi_{i}\right) Y_{\ell^{\prime}-m}\left(\theta_{j}, \phi_{j}\right)
$$

onde os YY são as harmónicas esféricas; todavia esta série tem o inconveniente de ser lentamente convergente para distâncias curtas, precisamente a zona em que a influência da orientação (anisotropia) deve ser mais forte. Outras dificuldades prendem-se com a noção de separação entre a parte repulsiva (cerne) e a parte atractiva (cauda) do potencial, e a assimilação da parte repulsiva com a forma efectiva da molécula, já que para moléculas poliatómicas o potencial global é uma função multidimensional da distância de separaçâo e dos ângulos de Euler. 


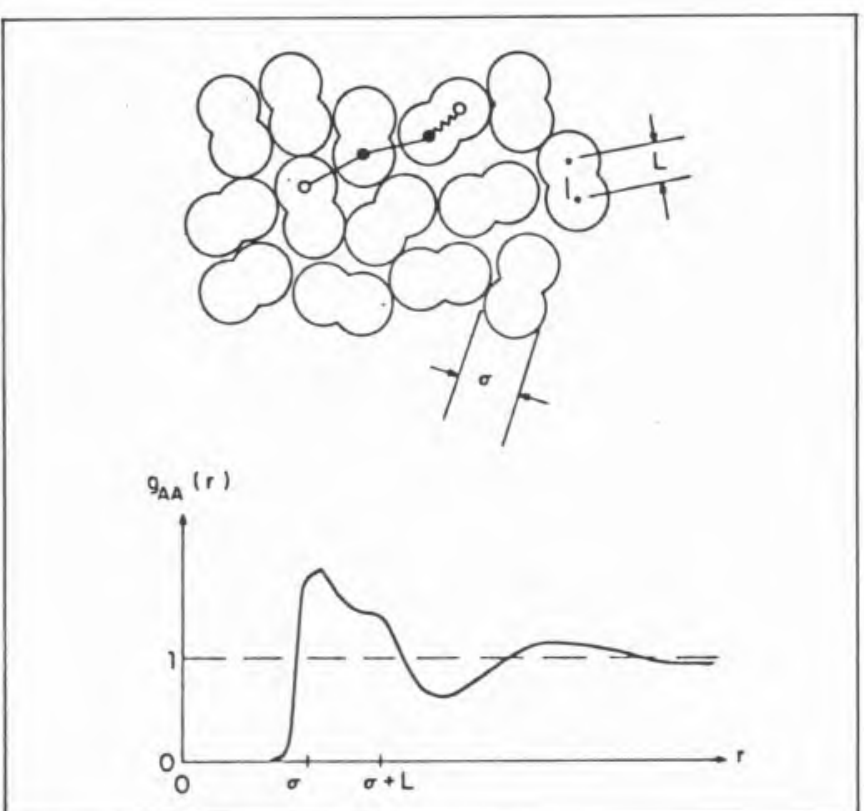

Fig. 8 - Função de disıribuição radial átomo-a-átomo nara um liquido de moléculas diatómicas $A,(\operatorname{Ref}$. 6)

Esıa figura é reproduzida, com autorização, do Annual Review of Physical Chemistry, Volume 29. 1978 by Anmual Review's Inc,

Uma alternativa consiste em construir funçōes de disıribuição átomo-a-átomo ou funçōes de distribuição de sítios ("site distribution functions"), cujo significado se torna evidente com a ajuda da figura 8 , que exemplifica o caso das moléculas diatómicas, compostas de esferas fundidas (diâmetro $\sigma$ e elongação L). Num estado denso como é o liquido, é provável que um átomo duma molécula esteja correlacionado com un átomı doutra, e a função de distribuição átomı-a-átomo $g_{11}(\mathrm{r})$ dá precisamente conta dessa correlação. O máximo de $\mathrm{g}_{\text {\} }(\mathrm{r}) \text { para } \mathrm{r} \sim \sigma \text { representa a distância de aproximação }$ mais provável para átomos de moléculas diferentes (pico intermolecular), enquanto o cotovelo para $r \backsim \sigma+\mathrm{L}$ resulta da presença inevitável do outro átomo da segunda molécula (pico inı ramolecular).

Chandler e os seus colaboradores usaram precisamente estas noçōes (coordenadas atómicas, funçôes de distribuição de sítios) para incorporar a estrutura molecular nos cálculos da mecânica estalística; desenvolveram assim um formalismo exacto, chamado 'série em cachos de interacçào de sítios' ("interaction site cluster series") onde os cachos derivam das sucessivas correlações de átomos inter e intramoleculares - dois a dois, três a três, etc. Este formalismo é passivel de várias aproximações, uma delas a equação integral RISM (iniciais de "reference interaction site model") que permite calcular as funções de distribuição átomo-a-átomo $\mathrm{g} \alpha \beta(\mathrm{r})$. Um trabalho recente de Chandler, Hsu e Streetı mostrou que a teoria RISM é, pelo menos, qualitativamente certa e está de acordo com os resultados obtidos com as simulações de Monte Carlo para moléculas diatómicas (homo e heteronucleares) ${ }^{12}$.

$\mathrm{O}$ conhecimento das funçôes de distribuiçào $\mathrm{g}_{\alpha} \beta^{(\mathrm{r})}$ permite chegar aos factores de estrutura $S(k)$, que se podem obter experimentalmente através de técnicas de difracçâo (raios XX, neutrões). $\mathrm{S}(\mathrm{k})$, onde.

$$
k=\frac{4 \Pi}{\lambda} \operatorname{sen} \theta
$$

mede a intensidade de radiação difundida segundo un certo ângulo em relação à intensidade incidente, e, de facto, $g(r)$ e $S(k)$ são transformadas de Fourier uma da outra. Na figura 9 comparam-se os valores experimentais

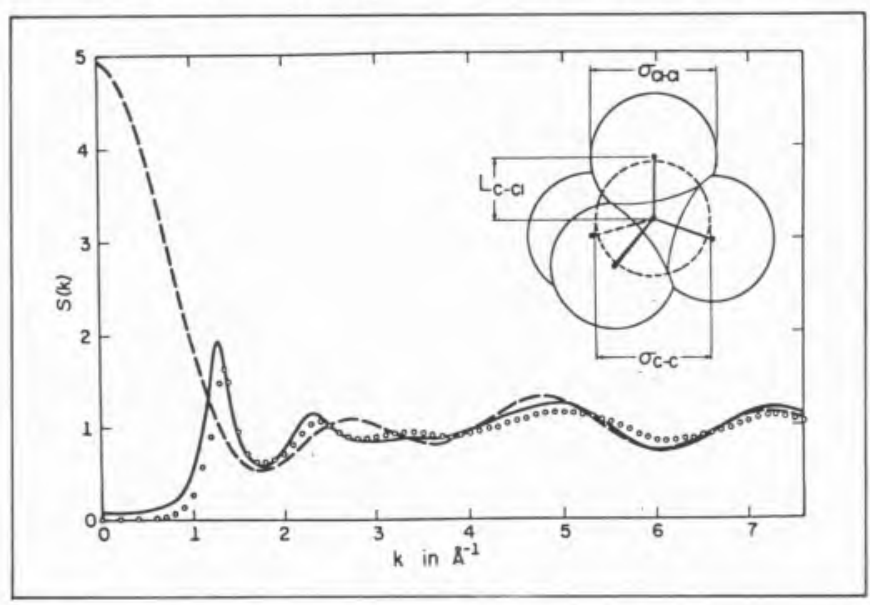

Fig. 9 - Factor de estrutura $S(k)$ do tel acloreto de carbono liquido. $o$, resultados experimentais; - , solução RISM; -..., gás perfeito. (Ref. 13). Esıa figura é reproduzida, com autorização, do Journal of Chemical Phvsics, Volume 61, pág. 5231. (c) 1974 by American Institute of Phvsics

obtidos para $\mathrm{S}(\mathrm{k})$ no caso do tetracloreto de carbono con a curva teórica obıida pelo RISM: a concordância é encorajadora ${ }^{13}$. Resultados análogos têm sido obtidos para o sulfureto de carbono $\mathrm{CS}_{2}$, benzeno $\mathrm{C}_{6} \mathrm{H}_{6}$, azoto, oxigénio, bromo e acetonit rilo $\mathrm{CH}_{3} \mathrm{CN}$.

$\mathrm{O}$ conhecimento da funçōes de distribuiçào $\mathrm{g}(\mathrm{r})$. quer por via teórica quer por via experimental, permite chegar às propriedades termodinâmicas, mas agora não podemos restringir-nos a modelos de corpos rígidos; temos de entrar em linha de conta com as forças atractivas que estào na origem da elevada densidade dos líquidos. As forças repulsivas permitem interpretar $\mathrm{g}(\mathrm{r})$ mas são insuficientes para explicar quantitativamente as propriedades termodinâmicas macroscópicas. O conhecimento combinado de $u(r)$ e $g(r)$ contém já Ioda a informação necessária para o cálculo das várias propriedades, nomeadamente a energia total (interna); para um liquido de moléculas esféricas é

$$
\mathrm{U}=\frac{3}{2} \mathrm{NkT}+2 \pi N \rho \int_{0}^{\infty} \mathrm{u}(\mathrm{r}) \mathrm{g}(\mathrm{r}) \mathrm{r}^{2} \mathrm{dr}
$$

O primeiro termo corresponde à contribuição da energia cinética e o segundo à da energia potencial, calculada na base da aditividade dos potenciais intermoleculares. Com efeito, $\rho \mathrm{g}(\mathrm{r})$ é a densidade numérica local, e o integral faz a soma das energias de interacção de todos os pares, quando a coroa esférica $4 \pi r^{2} \mathrm{dr}$ varre o espaço de integraçào.

O cálculo directo de integrais como o de (14) e difícil, entre outras razões porque $u(r)$ se torna infinito para $r=0$. O problema pode-se resolver aproximadamente, dividindo o potencial em duas partes - uma que contém a componente dura (repulsiva) do potencial e que constitui o potencial de referência, e a outra que contém a parte atractiva que é então tratada como uma perturbação da primeira. O sucesso destes métodos, chamados de perturbação, está na maneira como se faz tal separação: por um lado interessa que o potencial de referência dê origem a expressões facilmente calculáveis (por exemplo, o potencial de esferas rígidas); por outro interessa que o 
desenvolvimento em série de perturbação seja rapidamente convergente, o que só acontece se as estruturas do fluido real e do fluido de referência forem semelhantes.

$$
\mathrm{u}(\mathrm{r})=\mathrm{u}_{0}(\mathrm{r})+\gamma \mathrm{u}_{1}(\mathrm{r})
$$

$\mathrm{u}_{0}(\mathrm{r})$ é, pois o potencial intermolecular do liquido de referência e $u_{1}(r)$ a perturbação. $O$ parâmetro $\gamma$ caracteriza a intensidade da perturbação. As funçôes termodinâmicas são enıâo desenvolvidas emı série de potências de $\gamma$, em tormo de $\gamma=0$, fazendo no final $\gamma=1$. A energia de Helmholtz vem

$$
\frac{A}{N k T}=\frac{A}{N k T}+2 \pi \rho \int g_{0}(r) u_{1}(r) r^{2} d r(16)
$$

onde $A_{*}$ é a energia de Helmholtz do liquido de referência.

Tomando um líquido de esferas rígidas (de diâmetro variável com a temperatura) como referência. Barker c Henderson puderam calcular as propriedades termodinâmicas dum líquido com potencial realista, usando três termos do desenvolvimento em série ${ }^{14}-A_{\ldots} . A_{1}$ e $A$. No entanto, uma escolha mais feliz de $u_{i}(r)$ pode ievar a uma convergência mais rápida: é o que acontece na teoria das perturbacões WCA em que o potencial de referência contém toda a porçào que varia rapidamente con $r$, como se indica na figura 10 . Com este tipo de potencial

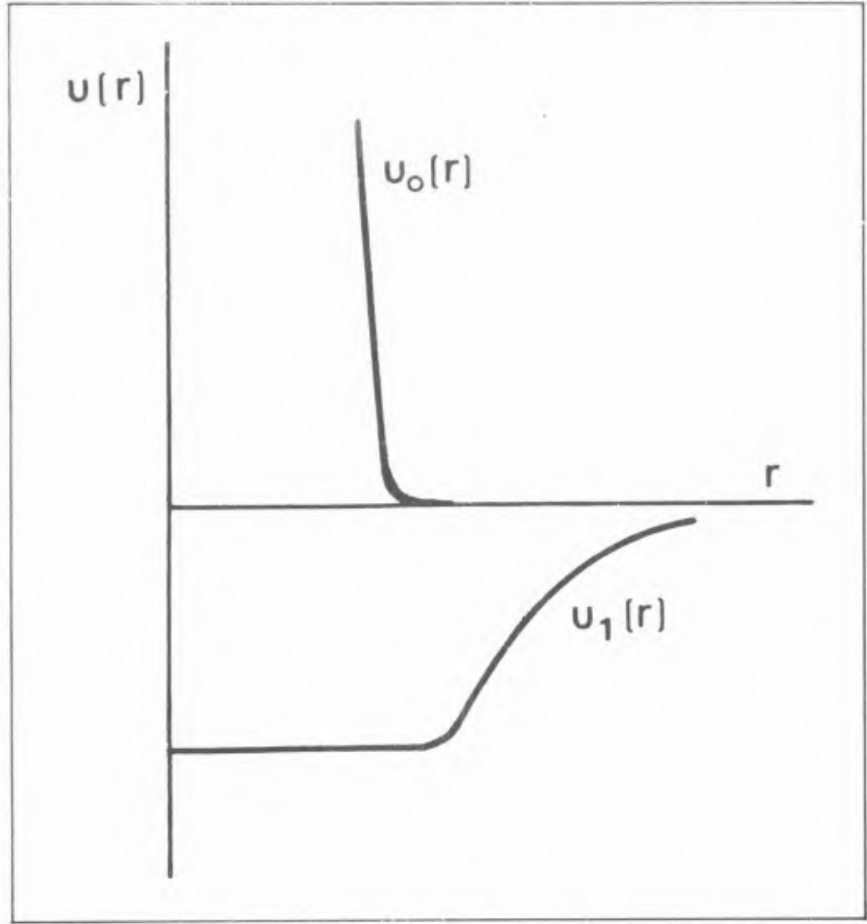

Fig. 10 - Polencial de referência $u_{n}(r)$ e polencial perturbador $u_{l}(r)$ na teoria WCA

de referência A pode ser calculado mais facilmente, pois a série converge com dois termos apenas.

E este problema da separação do potencial intermolecular em duas partes - uma de referência, variando rapidamente com as coordenadas, outra de perturbaçào, variando lentamente com $r$, que tem impedido o progresso da mecânica estatistica dos líquidos de moléculas dialómicas.

\section{5 - O Modelo Electrostático}

Nos números anteriores vimos como a forma da molécula medida através do respectivo potencial repulsivo, pode explicar muitas das propriedades dos liquidos, nomeadamente aquelas mais relacionadas com a estrutura. Um outro tipo de teorias procura interpretar o comportamento dos liquidos e suas misturas na base de forças atractivas, essencialmente de natureza electrostática. Com efeito, exceptuando o caso dos átomos dos gases raros, as moléculas têm dipolos e/ou multipolos (quadrupolo, octopolo, etc.) e é a interaccào entre estas entidades de natureza eléctrica que está na origem do modelo electrostático. Quanto maior for a simetria da molécula, mais clevado é o primeiro mulıipolo nào nulo. Assim, () cloreto de hidrogénio, $\mathrm{HCl}$, é dipolar, o azoto, N. é quadrupolar, o metano, $\mathrm{CH}_{4}$, é octopolar e o hexafluoreto de enxofre, $\mathrm{SF}_{h}$. é hexadecapolar.

Na sua versào mais simples, o modelo electrostático. desenvolvido por Gubbins e seus colaboradores ${ }^{15}$, considera as moléculas como pontuais e sobrepòe-lhes uma série de multipolos, o que confere anisotropia imediata ao potencial. De facto, os dipolos sào vectores e os quadrupolos, etc. são tensores, de modo que a energia de interaccão respectiva vai depender das orientaçòes relativas no espaço. Dizemos que as moléculas sào "pontuais" porque o porencial de referência escolhido é esfericamente smélrico, em geral do lipo I.ennard-Iones.

$$
\mathrm{u}\left(\mathrm{r}, \omega_{1}, \omega_{2}\right)=\mathrm{u}_{\mathrm{o}}(\mathrm{r})+\mathrm{u}^{\mathrm{a}}\left(\mathrm{r}, \omega_{1}, \omega_{2}\right)
$$

A parte anisotrópica $\mathrm{u}^{3}\left(\mathrm{r}, \omega_{i}, \omega_{2}\right)$ contém não só as forças de natureza electrostático-multipolar, como também as forças de indução, de dispersão e de sobreposição (repulsivas), isto é

$u^{a}\left(r, w_{1}, w_{2}\right)=u^{\text {mult }}+u^{\text {ind }}+u^{\text {disp }}+u^{\text {ov }}$

Em grande parte dos casos é o primeiro termo, $\mathrm{u}^{\text {mult }}$, que contribui significativamente para $\mathrm{u}^{\mathrm{a}}$, podendo alguns dos outros termos ser desprezados. Em princípio, em $\mathrm{u}^{\text {mult }}$ devem ser incluidas as várias interacções possíveis: dipolo-dipolo, dipolo-quadrupolo, etc. Se designarmoso dipolo por $\mu$, o quadrupolo por Q e o octopolo por $\Omega$, temos as seguintes contribuições de significado evidente

$$
u^{\text {mult }}=u^{\mu \mu}+u^{\mu Q}+u^{Q \mu}+u^{Q Q}+\ldots
$$

O valor relativo destas parcelas depende de caso para caso (intensidade dos momentos, etc.) mas, a menos que se trate dos gases raros ou de moléculas tetraédricas, a importância do quadrupolo é inegável. Quanto às forças de indução, fundamentalmente atractivas, elas resultam do facto do dipolo duma molécula induzir um dipolo na segunda, que por seu turno vai interactuar com o dipolo da primeira, etc.; fazem intervir a polarizabilidade $\alpha$, e temos

$$
u^{\text {ind }}=u^{\mu \alpha \mu}+u^{\mu \alpha Q}+u^{Q \alpha Q}+\ldots
$$

A energia de Helmholtz A é calculada a partir dum desenvolvimento em série de potências do potencial perturbador $\mathrm{u}^{\mathrm{a}}$, e a partir dela podem-se calcular as outras propriedades do sistema. 


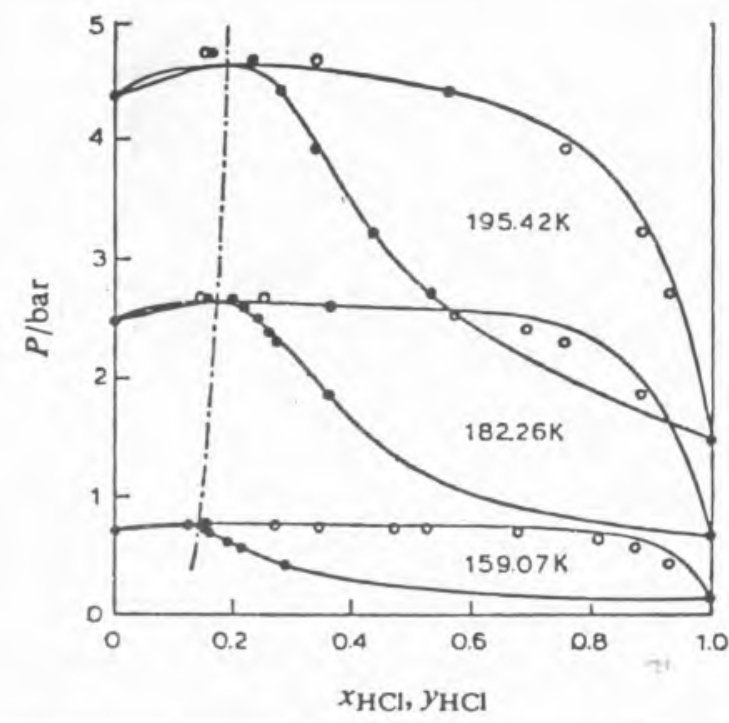

Fig. 11 - Equilibrio liquido-vapor para soluções liquidas HCl + Xe: $o$, pontos experimentais; - curvas teóricas. calculadas através do modelo electrostatico (Ref. 16)

Esıa figura é reproduzida, com autorização, do Journal of Chemical Society Faraday Transactions Parl I, Volume 74. pág. 904. $1978 \mathrm{by}$ The Chemical Socielv

Na figura 11 mostra-se a aplicaçào desta teoria ao caso do equilibrio liquido-vapor das misturas de xenon com cloreto de hidrogénio: os pontos são os resultados experimentais e as curvas foram traçadas teoricamente, independentemente daqueles ${ }^{16}$. O azeótropo é reproduzido, bem como o comportamento do sistema numa gama de baixas temperaturas, superior a $35 \mathrm{~K}$, e isto apesar das complicaçôes (ignoradas) devidas à existência das ligações por ponte de hidrogénio. E no entanto, algumas interrogações se levantam pois, como se sabe da electrostática, o desenvolvimento em série de multipolos não é válido para distâncias curtas, precisamente aquelas às quais estamos a aplicar o modelo electrostático. Por outro lado, qual o significado de grandezas como o momento dipolar, o momento quadrupolar, etc., no estado liquido, onde nâo há uma teoria satisfatória de dieléctricos para interpretar as propriedades de liquidos polares? O momento dipolar torna-se assim pouco mais do que um parâmetro ajustável.

Num artigo de revisão de 1977, Gubbins e Sıreeı afirmavam que «as forças multipolares têm um grande efeito tanto na estrutura como nas propriedades de equilibrio dos fluidos $)^{17}$, e um ano mais tarde Chandler argumentava, em artigo similar, as suas «sérias dúvidas sobre qualquer teoria que recorra largamente a simples interacçôes electrostáticas para interpretar a estrutura dum líquido»" ${ }^{6}$ acrescentando em seguida que "infelizmente uma grande porção de teorias do estado líquido baseia-se em tais modelos", , e referia os leitores para o artigo citado de Streett e Gubbins. Esta é uma das várias controvérsias que agitam hoje a físico-química dos líquidos. Mas as controvérsias são salutares fontes de progresso, e comparada com algumas do passado, a controvérsia forma-interacção multipolar não passa duma tempestade num copo de água... Por causa da lei da conservaçâo da energia Lord Kelvin e Tyndall cortaram relações, e Dühring perdeu a sua cátedra. Hoje espera-se dos químicos mais compostura, já que não se lhes pode pedir génio igual...

\section{6 - Final}

No domínio da física e química dos liquidos estamos, pois, ainda no princípio. E o princípio é o dos liquidos simples, constituidos por moléculas pequenas. Mas nâo devemos desanimar, ou pensar que por serem pequenas e simples estas moléculas carecem de interesse prático. É por serem pequenas e simples que as respectivas substâncias se apresentam, em condições normais, no estado gasoso - e o ar que respiramos tem oxigénio e azoto. O simples é, em mais de um sentido, o fundamental, e ainda há pouco o professor Fraústo da Silva nos lembrou, na sua lição plenária, que a química da vida é, afinal, a quimica dos elementos leves.

Falamos dos gases raros, do argon e do cripton. Muito se tem dito e escrito sobre os abusos do argon da parte dos estudiosos do estado líquido, a tal ponto de estes serem frequentemente acusados de sofrer duma doença nova a que se chamou argonite aguda... Mas a sonda Pioneer que há semanas nos revelou alguns segredos de Vénus, disse-nos que a sua atmosfera é cerca de quarenta vezes mais rica em argon do que a da Terra. Para os habitantes de Vénus a argonite seria, pois, uma doença mesmo séria!

Vi há semanas em Londres um filme fabuloso, pela mitologia que evoca, pela encenação, pela esperança e pelo humor, e também pelo uso fascinante de cristais (estado sólido, o espaço da memória) no planeta mítico onde tudo começa - SUPERMAN. Como sabem o Superhomem vem do planeta Cripton, e a sua missão na Terra é lutar pela Verdade e pela Justiça. Que se levante pois o primeiro que negue o valor do Cripton!

\section{BIBLIOGRAFIA}

[ 5] I.S. Row Inson, Chem, Soc. Revs., 7, 329 (1978).

[ 2] J.C.G. Cal ADo, M. Nunes da Ponte. V.A.M. Sonrese L.A.K. StAvitey, J. Chem. Thermodynamics, 10, 35 (1978).

[ 3] I.R. M.Dons। 1), in Statistical Mechanics - A Specialist Periodical Report, The Chemical Sociely, London, 1973.

[ 4] Rовғ Rт Musil, Der Mann ohne Eigenschafien, Rowohlı, Hamburg, 1952.

[5] H.C. Andirsen, Ann. Rev. Chem., 26, 145 (1975).

[6] D. ChANI)IIR, Ann. Rev. Phys. Chem... 29, 441(1978).

[ 7$]$ J.D. Wefks, D. Chanitifre H.C. Anisfrsen, J. Chem. Phys., 54, 5237 (1971).

[ 8 C.S. Hsu, D. Chandier e L.J. Lowden, Chem. Phys,, 14, $213(1976)$.

[ 9] C.A. Nieto de Castro, M. Nunes da Ponte e V.A. Meira So ArES, Técnica, n. ${ }^{\circ} 417,101$ (1972).

[10] J.A. BArker e D. Henderson, Rev. Mod. Phys., 48, 587 (1976).

[11] W.G. Hoover e F.H. ReE, J. Chem. Phys., 49, 3609(1968).

[12] D. Chandier, C.S. Hsu e W.B. Streeti, J. Chem. Phys., 66, 5231 (1977).

[13] L.J. Lowden e D. Chandi.er, J. Chem. Phys, 61, 5228 (1974).

[14] J.A. BArker e D. Henderson, J. Chem. Phys., 47, 2856 (1967); id. 4714.

[15] K.E. Gubiınse C.G. Gray, Mol. Phys., 23, 187 (1972); C.H. Twu, K.E. Gubisins e C.g. Gray, Mol. Phys., 29, 713 (1975). I

[166 J.C.G. Calado, C.G. Gray, K.E. Gubins, A.M.F. Pal avra, V.A.M. Sonres, L.A.K. Staveiey e C.-H. Twu, J.C.S. FArA. DAY I, 74, 893 (1978).

[17] W.B. Streett e K.E. Gubbins, Ann. Rev. Phys. Chem., 28, 373 (1977).

* Lição plenária proferida no 2. ${ }^{\circ}$ Encontro Nacional de Quimica. Porto, 5 Janeiro 1979. 